\title{
Long-term operation of a double phase LAr LEM Time Projection Chamber with a simplified anode and extraction-grid design
}

\author{
C. Cantini ${ }^{a}$, L. Epprecht ${ }^{a}$, A. Gendotti ${ }^{a}$, S. Horikawa ${ }^{a}$, S. Murphy ${ }^{a}$, G. Natterer $^{a}{ }$ \\ L. Periale ${ }^{a}$, F. Resnati ${ }^{a}$, A. Rubbia ${ }^{a *}$ F. Sergiampietri ${ }^{a, b}$, T. Viant ${ }^{a}$ and S. Wu ${ }^{a}$ \\ ${ }^{a}$ ETH Zurich, Institute for Particle Physics, \\ CH-8093 Zürich, Switzerland \\ ${ }^{b}$ INFN-Sezione di Pisa \\ 56127 Pisa, Italy \\ E-mail: Andre.Rubbiadeern.ch
}

\begin{abstract}
We report on the successful operation of a double phase Liquid Argon Large Electron Multiplier Time Projection Chamber (LAr LEM-TPC) equipped with two dimensional projective anodes with dimensions $10 \times 10 \mathrm{~cm}^{2}$, and with a maximum drift length of $21 \mathrm{~cm}$. The anodes were manufactured for the first time from a single multilayer printed circuit board (PCB). Various layouts of the readout views have been tested and optimised. In addition, the ionisation charge was efficiently extracted from the liquid to the gas phase with a single grid instead of two previously. We studied the response and the gain of the detector to cosmic muon tracks. To study long-term stability over several weeks, we continuously operated the chamber at fixed electric field settings. We reproducibly observe that after an initial decrease with a characteristic time of $\tau \approx 1.6$ days, the observed gain is stable. In 46 days of operation, a total of 14.6 million triggers have been collected at a stable effective gain of $G_{\infty} \sim 15$ corresponding to a signal-to-noise ratio $(S / N) \gtrsim$ 60 for minimum ionising tracks. During the full period, eight discharges across the LEM were observed. A maximum effective gain of 90 was also observed, corresponding to a signal-to-noise ratio $(S / N) \gtrsim 400$ for minimum ionising tracks, or $S / N \approx 10$ for an energy deposition of $15 \mathrm{keV}$ on a single readout channel.
\end{abstract}

KEYWORDS: liquid Argon; low capacitance readout; TPC; double phase; charge extraction; tracking.

${ }^{*}$ Corresponding author. 


\section{Contents}

1. Introduction 11

2. The experimental setup 2]

2.1 New 2D views, low-capacitance anodes

2.2. Single extraction grid

3. Operation and performance assessment

3.1 Straight track reconstruction

3.2 Definition of the stable effective gain 9

4. Results with anode A and stability of the gain

5. Results with anodes B, C and D

6. Summary of results 16

7. Conclusion 18

\section{Introduction}

The liquid Argon time projection chamber (LAr-TPC) [1] is a charge imaging detector which allows to reconstruct tracks in three dimensions as well as the locally deposited energy. In this context, the Giant Liquid Argon Charge Imaging ExpeRiment (GLACIER) is a concept proposed

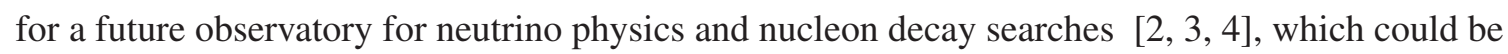
scalable up to gigantic masses. This design is contemplated for $20 \mathrm{kton}$ and $50 \mathrm{kton}$ detectors in the LBNO Expression of Interest submitted to CERN [5].

The key and innovative feature of the GLACIER design is the double phase LEM-TPC oper-

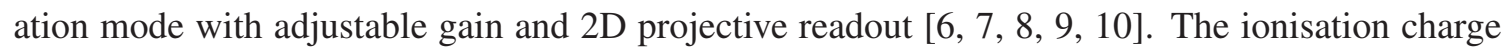
is extracted to the Argon gas phase where it is amplified by a Large Electron Multiplier (LEM) which triggers Townsend multiplication in the high electric field regions in the LEM holes [10, 11]. The charge is collected and recorded on a two-dimensional and segmented anode. This principle has two main advantages: 1. the gain in the LEM is adjustable, i.e. the signal amplitude can be optimised for $>99 \%$ hit reconstruction efficiency, and 2. the signals collected on the two readout views are unipolar and symmetric which facilitates the event reconstruction. In addition to the ionisation charge, scintillation light is emitted by excited Argon diatomic molecules (excimers) (see e.g. Ref. [12]). The detection of the scintillation light is fast, thus providing the event time reference $T_{0}$. For recent reviews on cryogenic detectors with electron avalanching see e.g. Ref. [13], [14]. 
We have described the successful operation of this kind of detector in previous publications (see for instance Ref. [6, 团]). In this paper, we report on a simplified design of two dimensional projective readout anodes, manufactured from a single multilayer printed circuit board (PCB), leading to a reduction of the electrical capacitance of its electrodes. To further simplify the design, we also decided to extract the drifting electrons from the liquid to the gas by means of a single grid placed just below the liquid surface. These developments have been carried out in view of the extrapolation of this technique to large surfaces (typically one square meter), as needed for the GLACIER design. The new readouts were manufactured and tested on a prototype chamber located at CERN. The response of the chamber in terms of effective gain, signal-to-noise ratio and energy resolution was checked for different settings of the electric fields across each stage. Furthermore, a stable operation of the TPC allowed us to retrieve a data set of approximately 14.6 million events. The large statistics enabled us to study the gain stability over longer periods.

\section{The experimental setup}

The so-called " 3 liter" setup is a double phase LAr LEM TPC [10, [15, 16] consisting of a $21 \mathrm{~cm}$ long drift volume and a $10 \times 10 \mathrm{~cm}^{2}$ area. Even if of modest size, it is very useful for testing new ideas with a rapid turn-around. The setup has been developed by the ETHZ group during the last four years and various charge readout devices were reliably operated with it, as reported elsewhere [0, 8]. A picture of the chamber and the schematics of the setup are presented in Figure [1. The ionisation electrons drift vertically towards the liquid surface by means of a homogenous electric field (of strength typ. $500 \mathrm{~V} / \mathrm{cm}$ ) provided by field shapers spaced out every $5 \mathrm{~mm}$. A larger electric field of about $2 \mathrm{kV} / \mathrm{cm}$ is applied at the vicinity of the liquid Argon surface to efficiently extract the drifting charges to the vapour phase. The extraction field is confined in a $10 \mathrm{~mm}$ region between the Large Electron Multiplier (LEM) and a stainless steel etched mesh placed in the liquid (see Section 2.2). The amplification and readout of the charge is then performed by the LEM and anode layers. The $1 \mathrm{~mm}$ thick LEM used in this setup has about 16'000 holes of $500 \mu \mathrm{m}$ spaced at a distance of $800 \mu \mathrm{m}$ from each other with a dielectric rim of $50 \mu \mathrm{m}$, and an active area which matches that of the readout. An electric field of at least $30 \mathrm{kV} / \mathrm{cm}$ is applied across the LEM in order to obtain charge amplification. The electrons are collected on the anode placed $2 \mathrm{~mm}$ above the LEM. The digitisation and data acquisition is performed by the specially developed CAEN SY2791 readout system (see Ref. [6]). The detector is also equipped with a Hamamatsu R11065 photomultiplier tube (PMT) placed below the cathode grid and made sensitive to the VUV scintillation light with the wavelength shifter tetraphenylbutadiene (TPB) [12]. The PMT provides the event $T_{0}$ and trigger by detecting the prompt photons from the liquid Argon scintillation.

In order to keep the 3 liter chamber at thermal equilibrium, it is fully immersed in an open bath filled with liquid argon. The bath evaporates to open air, the pressure and temperature are rather stable until the level of the liquid argon bath goes below the top flange of the 3 liter chamber. At this point, the heat input increases and the pressure inside the chamber rises. The pressure inside the detector increases until the operator refills the liquid argon bath. This is done periodically, on a daily basis. In addition, the changes of atmospheric pressure are also reflected on the temperature and pressure of the setup since the bath is open. These pressure changes will affect the amplification in the LEM as will be shown in Section $\Psi$, however this effect on the gain can be corrected for during 

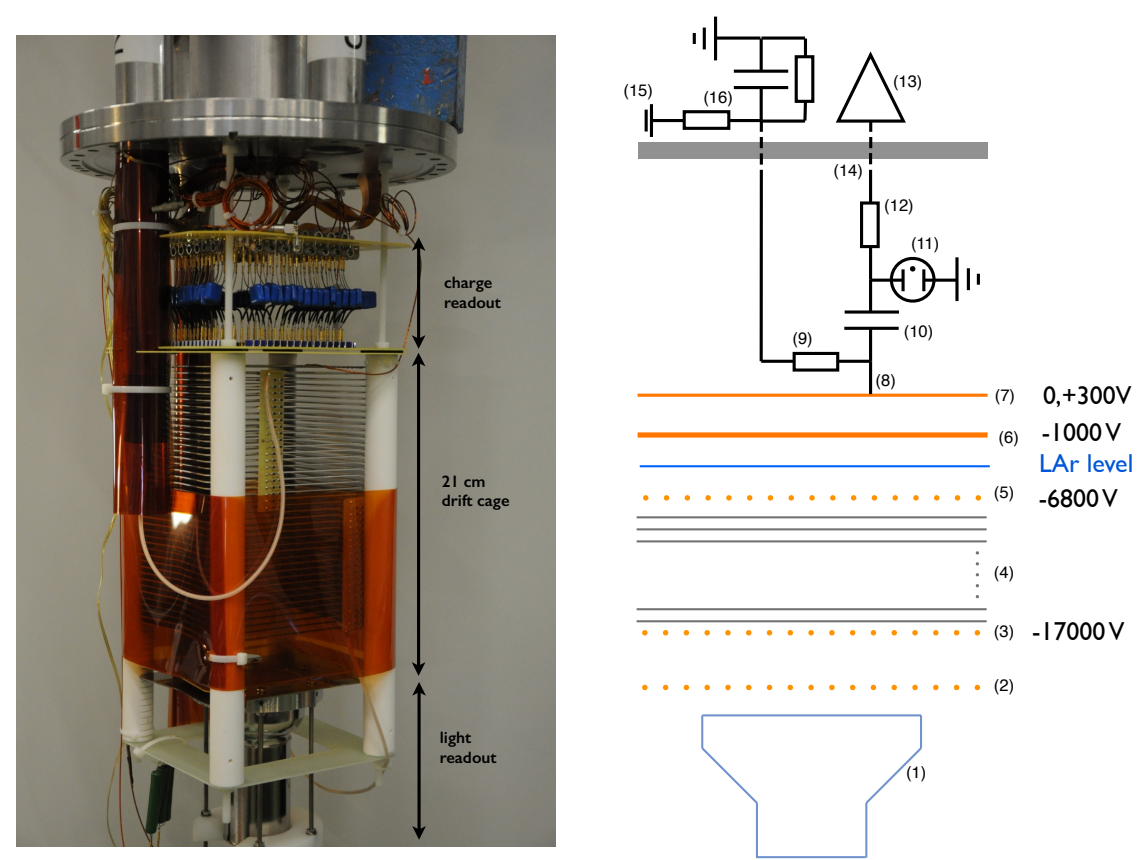

Figure 1. Left: Picture of the ETHZ 3 liter double phase LEM-TPC. Right: schematic representation of the detector showing the PMT (1) and its protection grid (2), the cathode (3), the field shaping electrodes (4), the single extraction grid (5), the LEM (6), the two views low capacitance anode (7), the connection to the strips (8), the $500 \mathrm{M} \Omega$ high voltage resistor (9), $270 \mathrm{pF}$ high voltage decoupling capacitor (10), the surge arrester (11), the current limiting $33 \Omega$ resistor (12), the preamplifier (13), the flange and the electrical feedthroughs (14), the high voltage power supply (15), the low pass filter and the resistor divider (16). Elements from (8) to (13) are replicated for each readout channel. The voltages indicated on the right are those applied across each stage for the LEM operated at $33 \mathrm{kV} / \mathrm{cm}$.

offline data-analysis. To avoid such effects in future detectors, it is straightforward to regulate the pressure avoiding open bath, as was for example the case in our 200 liter setup [6], where the pressure was stabilised to \pm 1 mbar.

Before filling the inner vessel with (pure) liquid Argon, the volume is evacuated to residual pressures below $5 \times 10^{-6}$ mbar to favor the outgassing of the materials. During the filling the liquid Argon is passed through an activated copper and zeolite powder cartridge which traps the oxygen and moisture impurities from the liquid. The filling is performed slowly, until the level of the liquid is precisely adjusted inbetween the grid and the LEM. To maintain the purity for long periods, the liquid Argon is evaporated and pushed by a bellows pump through a commercial SAES getter ${ }^{1}$. The gas Argon is then condensed through a serpentine immersed in a liquid Argon open bath, and again reintroduced as liquid inside the detector. The liquid Argon purity is monitored throughout the data taking period by measuring the lifetime of the drifting electrons.

\subsection{New 2D views, low-capacitance anodes}

The anode consists of two orthogonal sets of strips (views) that provide reconstruction of the $x$

\footnotetext{
$1_{\text {WwW.saesgetters.com }}$
} 
and $y$ coordinates of the ionising event. It is designed in such a way that the amplified charge is (equally) shared and collected on both views. This feature simplifies the extraction of the signal waveforms and eases the event reconstruction process.

In our previous designs [ [7, 15], symmetric charge sharing was achieved by producing anodes which consisted of two orthogonal sets of copper strips interleaved with a $50 \mu \mathrm{m}$ thick Kapton insulator. Those anodes, referred to hereafter as Kapton foil anodes, had a measured capacitance per unit length as high as $\sim 600 \mathrm{pF} / \mathrm{m}$ due the proximity of the copper strips. As described in Ref. [G], the measured RMS value of the intrinsic equivalent input noise charge (ENC) for a capacity $C$ at the input of our preamplifiers is $470 \pm 30 e^{-}$for $C=10 \mathrm{pF}, 580 \pm 30 e^{-}$for $C=92 \mathrm{pF}, 770 \pm 30$ $e^{-}$for $C=210 \mathrm{pF}$, and $1420 \pm 30 e^{-}$for $C=480 \mathrm{pF}$. For our typical mode of operation, the ENC should be below $1000 e^{-}$, implying an upper bound on the input capacitance at $\sim 400 \mathrm{pF}$. Hence, the Kapton foil anodes are hardly compatible with an area larger than a square-meter, without a degradation of the noise performance.

In light of those requirements, we designed new types of anodes with both views printed on a single Printed Circuit Board (PCB) layer. Such anodes can be easily produced using photolithographic etching techniques (See Figure 2). Four different types of anodes labelled A,B, C and D (see Figures 3) were commercially manufactured ${ }^{2}$. Their dimensions and properties are listed in Table [1. The $3 \mathrm{~mm}$ strips consist of interconnected gold plated copper tracks. The anodes mainly differ by the number of tracks per readout strip.

Anode A is not perfectly $x-y$ symmetric since for one view the connection between the tracks is printed on the readout side while for the other view the tracks are linked on the other side by a via. Anodes B, C and D on the other hand are designed to be fully symmetric for both views. Since both views are linked by vias, they are printed on a multilayer PCB. In the case of anode $\mathrm{B}$, the track pitch matches the readout pitch of $3 \mathrm{~mm}$ and therefore it is the anode that offers the lowest capacitance per unit length. However with such a coarse track pitch, the charge collected from an ionising track may not be equally shared between both views (as will be shown later). In that respect anodes $\mathrm{C}$ and $\mathrm{D}$ were also tested. They are similar in design to anode $\mathrm{A}$ but the inter-connections between the pads are symmetric for both views. Anode $\mathrm{C}$ has three copper tracks per strip and therefore has similar capacitance per unit length as anode A. Anode D has two tracks per strip and hence offers a lower capacitance. The measured capacitance per unit length for each anode is reported in Table 11. Compared to the Kapton foil anode, the electrical capacitance per channel of the new anodes is reduced by a factor 2 to 6 .

\subsection{Single extraction grid}

Electric fields of the order of $2 \mathrm{kV} / \mathrm{cm}$ in liquid are needed to efficiently extract the electrons from the liquid to vapor phase. In our previous setups [7] the extraction field region was confined between two parallel $100 \mu \mathrm{m}$ diameter wire grids placed with a gap of $10 \mathrm{~mm}$ across the liquidvapor interface. Electrons may however be collected on the top grid due to their diffusion in the gas phase, and a potential misalignment of the two grids leads to the consequence that the grid system is not fully transparent for the drifting electrons. To resolve these issues, we tested extraction

\footnotetext{
${ }^{2}$ Anodes A, C and D were produced by Multi-CB (www.multi-circuit-boards.eu) and anode B by ELTOS (www.eltos.com)
} 


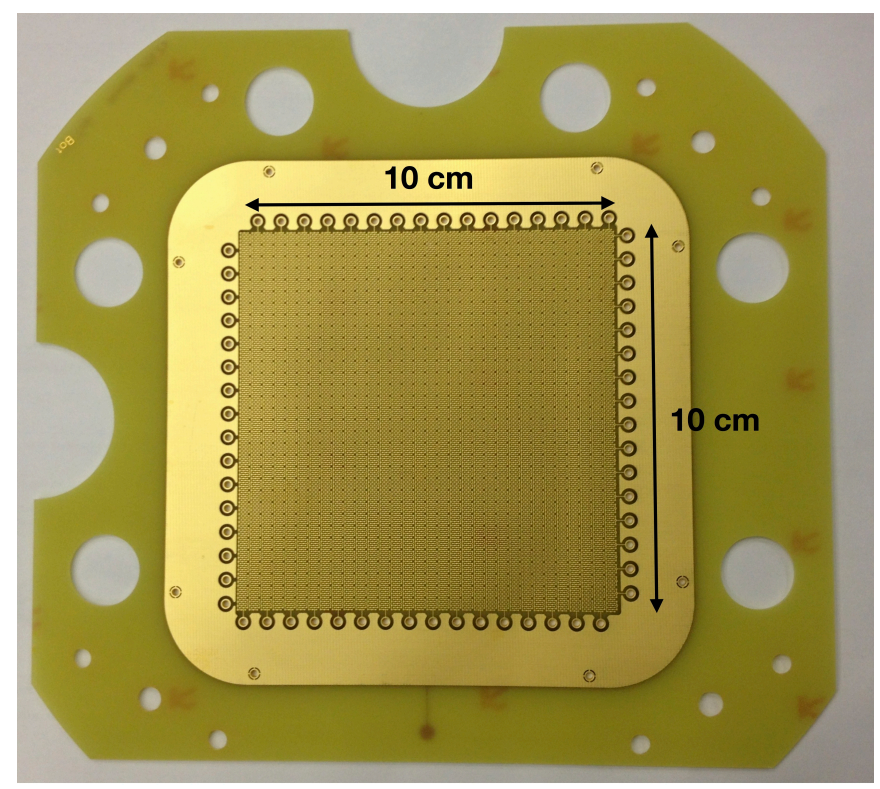

Figure 2. Picture of one of the 10x10 $\mathrm{cm}^{2}$ multilayer PCB anodes (here anode A) tested in our chamber.

\begin{tabular}{lllll}
\hline Parameter & Anode A & Anode B & Anode C & Anode D \\
\hline Copper thickness $(\mu \mathrm{m})$ & 35 & 35 & 35 & 35 \\
Epoxy thickness $(\mathrm{mm})$ & 1.5 & 5.1 & 1.5 & 1.5 \\
Readout pitch $(\mathrm{mm})$ & 3 & 3.125 & 3 & 3 \\
Track pitch $(\mathrm{mm})$ & 1 & 3 & 1 & 1.5 \\
Track width $(\mathrm{mm})$ & 0.1 & 0.3 & 0.1 & 0.1 \\
Pad diameter $(\mathrm{mm})$ & 0.4 & 1 & 0.4 & 0.4 \\
Via diameter $(\mathrm{mm})$ & 0.2 & 0.3 & 0.2 & 0.2 \\
Measured capacitance $(\mathrm{pF} / \mathrm{m})$ & 230 & 100 & 260 & 140 \\
\hline
\end{tabular}

Table 1. Characteristics of the $2 \mathrm{D}$ PCB anodes. For anodes $\mathrm{C}$ and $\mathrm{D}$ the capacitance was measured from a full $50 \times 10 \mathrm{~cm}^{2} \mathrm{PCB}$, while for anodes A and B the measurements are extrapolated from the $10 \times 10 \mathrm{~cm}^{2}$ boards. The copper thickness corresponds to the approximate layer after the final etching phase.

with a single grid placed in the liquid. In this configuration the LEM is positioned just above the liquid surface and the extraction field is directly provided by the LEM-grid system over the 

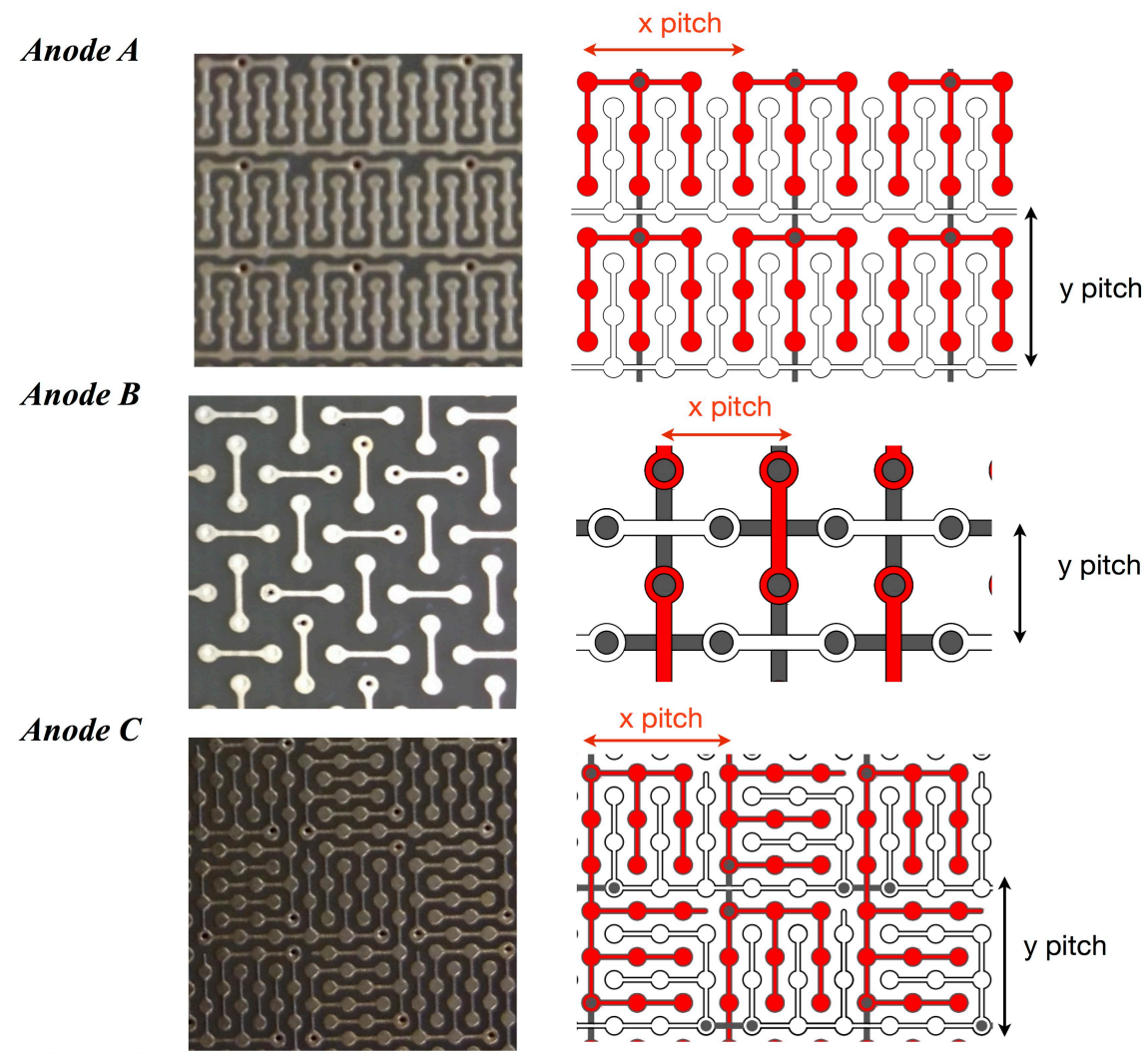

Anode D
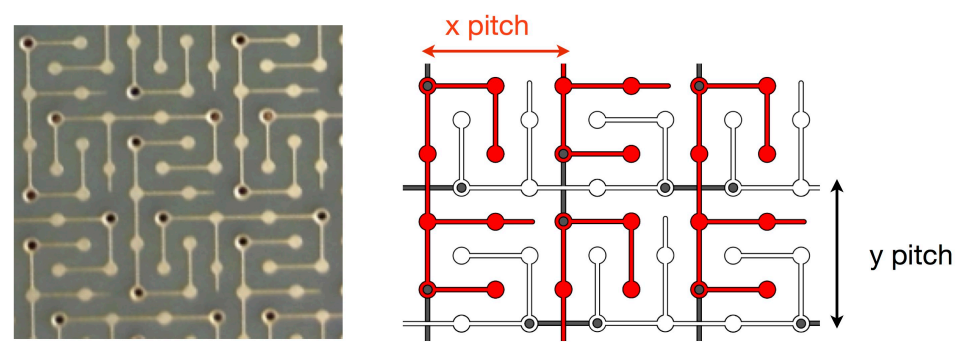

Figure 3. Close up pictures of the $2 \mathrm{D}$ anode prototypes showing the copper track pattern that allows a 2 view readout on the same circuit board. Schematics explaining the interconnections between both views are shown on the right. The $3 \mathrm{~mm}$ readout pitches are indicated by arrows. View 0 is filled in red and view 1 in white.

$10 \mathrm{~mm}$ distance. Electrostatic calculations of the field lines at the interface are shown in Figure 4 for a double- and single-grid extraction. In the former, the spatial distribution of the charge is maintained in the gas since the electrons are first focalised between the grids and de-focalised to the original pattern after the second grid. In the single grid geometry, the charges stay bunched as they arrive to the LEM and the size of the bunches is given by the wire pitch. Opting for a single 


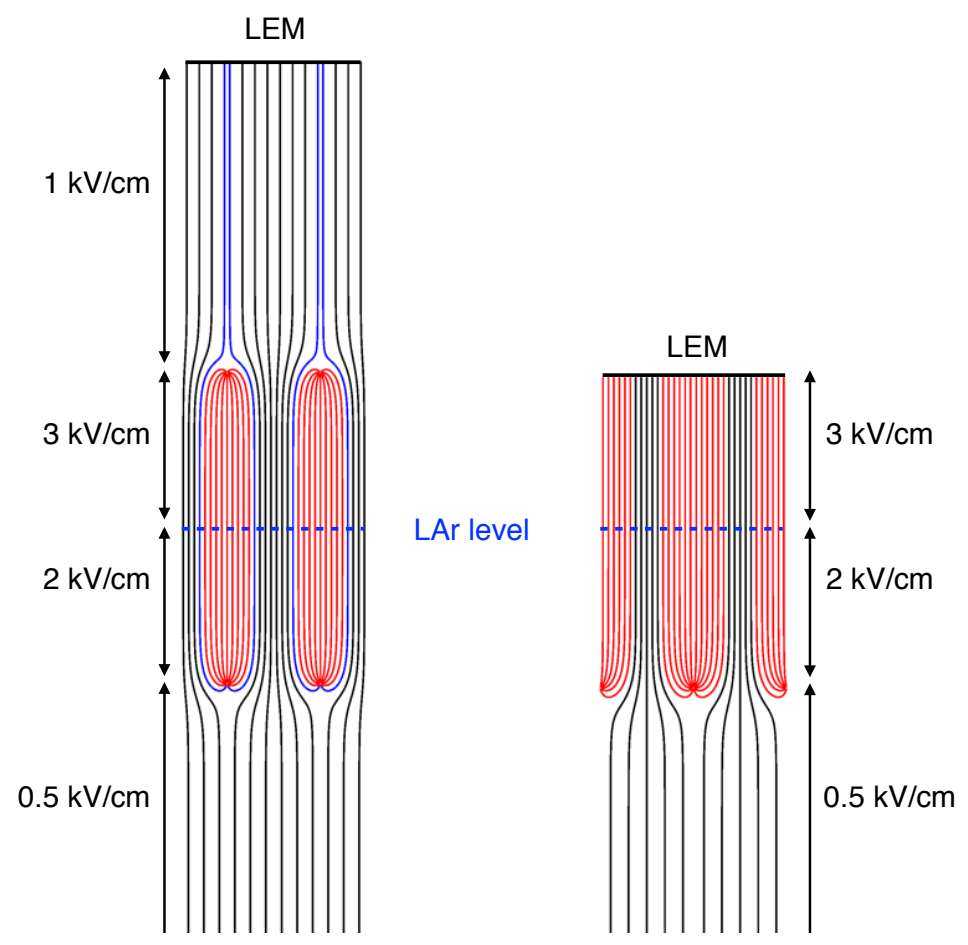

Figure 4. Electric field lines at the liquid-gas interface for the double grid (left) and single grid (right) configuration. The field lines followed by the drifting charge are drawn in black.

grid system offers the extra advantage of simplifying the overall design and construction of the readout system. Furthermore it reduces the anode-grid distance and lowers the absolute value of the required high voltage.

In our test, the extraction plane was constructed with a $1.5 \mathrm{~mm}$ pitch mesh, etched from a stainless steel plate. The strips had a width of $150 \mu \mathrm{m}$. With this configuration and with the electric fields indicated in Figure 4 , all electrons are effectively transported towards the LEM.

\section{Operation and performance assessment}

The cosmic data collected with each anode is summarised in Table Z. To study long-term stability, we decided to operate the chamber equipped with the different anodes at an asymptotically stable gain of $G_{\infty} \sim 15$ (see Section 3.2 for the definition of $G_{\infty}$ ). The electric field applied across each stage that were set for the long term operation are reported in Table 3. We could verify that under this condition, the setup is very stable. In a total 46 days of operation, a comprehensive set of 14.6 million triggers has been collected and only 8 discharges observed. Further tests will be performed in the future to assess the long-term stability at higher gains. The results obtained with anode A are reported in Section 4 . The comparison between data collected with other anode geometries is discussed at the end of the paper in Section 5 .

\subsection{Straight track reconstruction}

Cosmic muons that cross the chamber are minimum ionising particles (MIPs) depositing a known 


\begin{tabular}{cccccc}
\hline & \multicolumn{3}{c}{ data taking period } & number of triggers & number of discharges \\
\cline { 2 - 4 } & start & stop & days running & & \\
\hline anode A & 11-Apr & 16-May & 20 & $7.5 \mathrm{M}$ & 6 \\
anode B & 16-July & 29-July & 12 & $4.2 \mathrm{M}$ & 2 \\
anode C & 27-Aug & 02-Sept & 7 & $1.4 \mathrm{M}$ & 0 \\
anode D & 15-Oct & 21-Oct & 7 & $1.5 \mathrm{M}$ & 0 \\
\hline
\end{tabular}

Table 2. Data taking periods for each anode.

\begin{tabular}{lcc}
\hline & distance $[\mathrm{mm}]$ & nominal electric field $[\mathrm{kV} / \mathrm{cm}]$ \\
\hline Induction (anode-LEM) & 2 & 5 \\
Amplification (LEM) & 1 & 33 \\
Extraction (LEM-grid) & 10 & 2 \\
Drift field (grid-cathode) & 210 & 0.5 \\
\hline
\end{tabular}

Table 3. Electric field configuration for the long-term operation.

amount of energy of about $2.1 \mathrm{MeV} / \mathrm{cm}$ and are identifiable by events with a single "straight track" (plus possibly accompanying delta-rays). Such events can be used to characterise the detector in terms of free electron lifetime and amplification. In particular the measurement of the electron lifetime $\tau_{e}$ is retrieved by fitting the average energy loss of the reconstructed tracks as a function of the drift time $\left(t_{\text {drift }}\right)$ with the exponential law $e^{-t_{\text {drift }}} / \tau_{e}$.

The same offline procedure is applied to each set of data listed in Table [, and allows for a direct comparison of the anode performance. A typical cosmic track event collected with the electric fields listed in Table 3, is shown in Figure 5. The channel number and the drift time are plotted on the $x$ and $y$ axis respectively and the gray scale is proportional to the signal amplitude. The 3D reconstruction of the muon tracks from the digitised raw waveforms is accomplished with the Qscan software package [18]. A set of algorithms is consecutively applied to filter coherent pick up noise that could not be removed online, remove discrete noise frequencies and perform pedestal subtraction. Physical hits are subsequently extracted from the signal waveforms by means of a standard threshold discrimination algorithm. Once calibrated, the hit integral is a direct measurement of the local energy deposition of the ionisation track in the liquid Argon medium. The next step consists in identifying tracks by clustering and fitting neighbouring hits. A tree finding algorithm [17] efficiently finds tracks by searching for the longest consecutive set of hits. The connected hits are then fitted with a straight line and the $z$ coordinate of the 3D track is computed by matching the end point drift times of the tracks from both views. The 3D reconstruction allows to retrieve the length of the track on each strip of view 0 and view $1\left(\Delta s_{0}\right.$ and $\left.\Delta s_{1}\right)$, along with the charge collected on the corresponding channels, $\Delta Q_{0}$ and $\Delta Q_{1}$. The ratios $\Delta Q_{0} / \Delta s_{0}$ and $\Delta Q_{1} / \Delta s_{1}$ which are proportional to the energy locally deposited by the track in liquid Argon per unit length, are the relevant quantities used to estimate the gain of the chamber (see Eq. 3.1). In addition to 

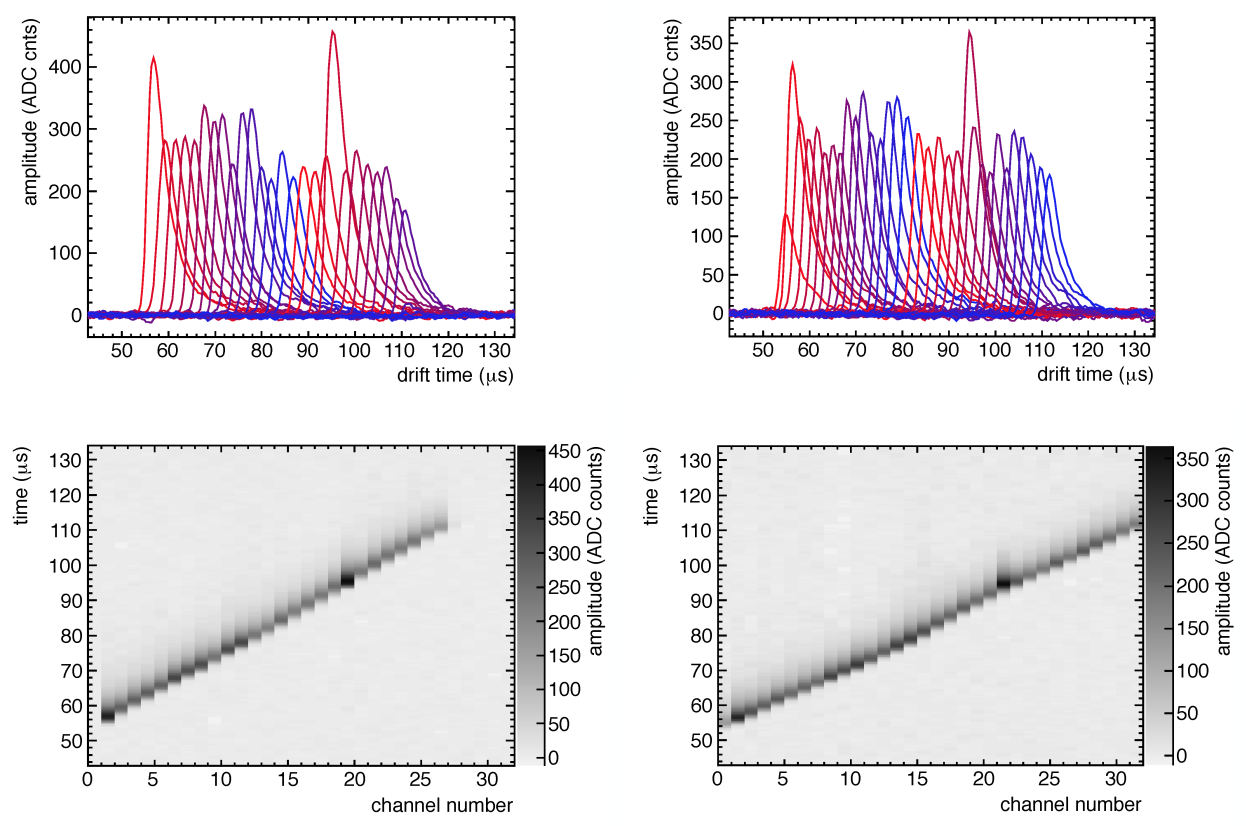

Figure 5. Event display of a cosmic track. (top): the raw waveforms showing the amplitude of the signals on both views (different colours for different readout channels). (bottom): drift time versus channel number of the reconstructed hits. This event was collected with anode A and the nominal values of the electric field settings described in the text.

making a selection on the goodness of the linear fit, only tracks that cross the entire detector were retained by applying a selection criteria on the endpoints of the reconstructed 3D tracks. Examples of $3 \mathrm{D}$ reconstructed hits and corresponding tracks are displayed in Figure 6 .

\subsection{Definition of the stable effective gain}

As explained in Ref. [Z], we define the effective gain $G_{\text {eff }}$ of the device as the ratio of the measured charge (corrected for the finite drift electron lifetime) collected on both views to the predicted charge deposition of an ionising particle. Under the assumption that only MIP events are present in our selected sample, the average charge deposition along a track, predicted by the Bethe-Bloch formula and accounting for electron-ion recombination [19] is $\langle\Delta Q / \Delta s\rangle_{M I P}=10 \mathrm{fC} / \mathrm{cm}$. The effective gain is hence defined as

$$
G_{e f f}=\frac{\left\langle\Delta Q_{0} / \Delta s_{0}\right\rangle+\left\langle\Delta Q_{1} / \Delta s_{1}\right\rangle}{\langle\Delta Q / \Delta s\rangle_{M I P}}
$$

where the indices correspond to view 0 and 1.

The measured $G_{\text {eff }}$ takes into account the charge multiplication in the LEM holes, as well as potential charge reduction from the liquid-vapour extraction efficiency and from the electrostatic transparency of the grid and the LEM. For amplification in holes across a LEM of thickness $d$ with the nominal electric field $E_{0}=V / d$, it is convenient to express $G_{\text {eff }}$ with the function [प]:

$$
G_{e f f}\left(E_{0}, \rho, t\right) \equiv \mathscr{T} e^{\alpha\left(\rho, \kappa E_{0}\right) x} \times \mathscr{C}(t)
$$



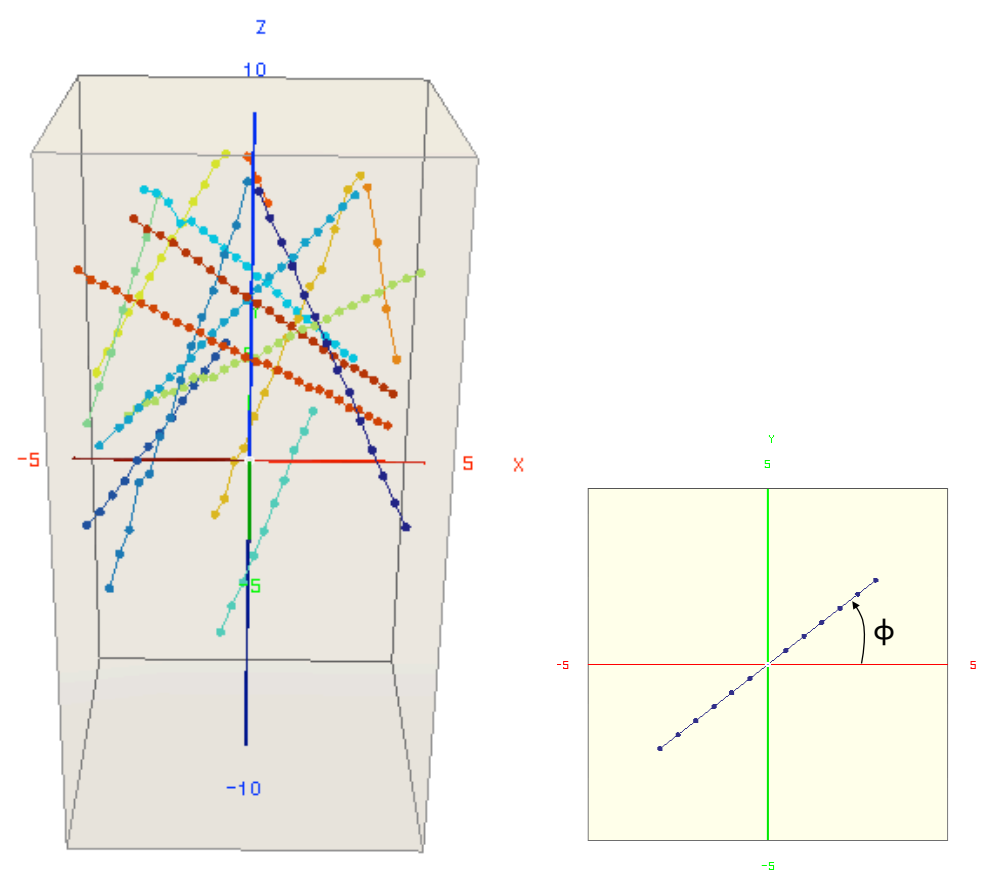

Figure 6. Left: reconstructed $3 \mathrm{D}$ hits and tracks from crossing cosmic rays. The $z$ coordinate is that of the drift and the $x-y$ coordinates are given by both views of the anode. Right: top view $(x-y$ plane $)$ of the detector.

where $\mathscr{T}$ is the transparency; $\alpha(\rho, E)$ is the first Townsend ionisation coefficient for the electric field $E$ and density $\rho ; x$ denotes the effective amplification length which can be geometrically related to the length of the field plateau along the hole; and $\mathscr{C}(t)$ represents any time variation of the gain. Electrostatic calculations of the LEM-hole geometry give a maximum field in the hole which is lower than the naive $V / d$, consistent with a value of $\kappa=0.95$ and an effective length in the range of $0.7 \mathrm{~mm}$ for a $1 \mathrm{~mm}$ thick LEM. The generalised form of the first Townsend coefficient as a function of the medium density $\rho$ and the electric field $E$ can be approximated by [20]:

$$
\alpha(\rho, E)=A \rho e^{-B \rho / E}
$$

where $A$ and $B$ are parameters depending on the gas. A fit to the electric field dependence of the Townsend coefficient in the range between 20 and $40 \mathrm{kV} / \mathrm{cm}$ predicted by MAGBOLTZ [2]] calculations, gives $A \rho=(3160 \pm 90) \mathrm{cm}^{-1}$ and $B \rho=(136.4 \pm 1.0) \mathrm{kV} / \mathrm{cm}$ for pure argon at $87 \mathrm{~K}$ and 1 bar.

For reasons explained later in Section $\$$, the long-term behavior of our setup is consistent with an electrostatic charging up of the dielectric medium of the LEM during operation, which reaches a stable condition after a characteristic time for charging-up. After setting a particular electric field configuration, an initial reduction of $G_{e f f}$ is observed, which is reproducible and characteristic of the setup. Empirically we define the "stable effective gain" $G_{\infty}$ as:

$$
G_{e f f}(t) \simeq G_{\infty} \times \frac{1}{\left(1-e^{-t / \tau}\right)}
$$

where $\tau$ is the characteristic charging-up time, to be determined from the data. 


\section{Results with anode A and stability of the gain}

To quantify the long term stability of the gain, we mounted the chamber with a given anode and observed the evolution of the gain over a designated period. The duration of the data taking with each anode is listed in Table 2]. The run with anode A was the longest and is therefore described in this section as example.

The data-taking with anode A lasted for more than a month at a constant event trigger rate of about $5 \mathrm{~Hz}$. During this period data was collected almost continuously, without any changes in the settings and without opening the chamber. The data acquisition was interrupted three times for a few days in order to purify the liquid Argon. The measured level of impurities in the Argon (in Oxygen equivalent) estimated from the electron lifetime $\tau_{e}$, is presented in Figure 7 over the full period of data-taking. At the beginning of the detector operation the electron lifetime was around $360 \mu \mathrm{s}$, which corresponds to an oxygen equivalent impurity of $\sim 1 \mathrm{ppb}$. During the period of datataking, the gas recirculation is switched off, and a degradation of the purity of about 1.4 ppb per day is observed. The origin of the Argon contamination is interpreted as stemming from outgassing of the surfaces of the various components of the chamber. Once the liquid Argon contamination reaches a level of about $10 \mathrm{ppb}$ oxygen equivalent the liquid Argon is purified for a period of 3-5 days.

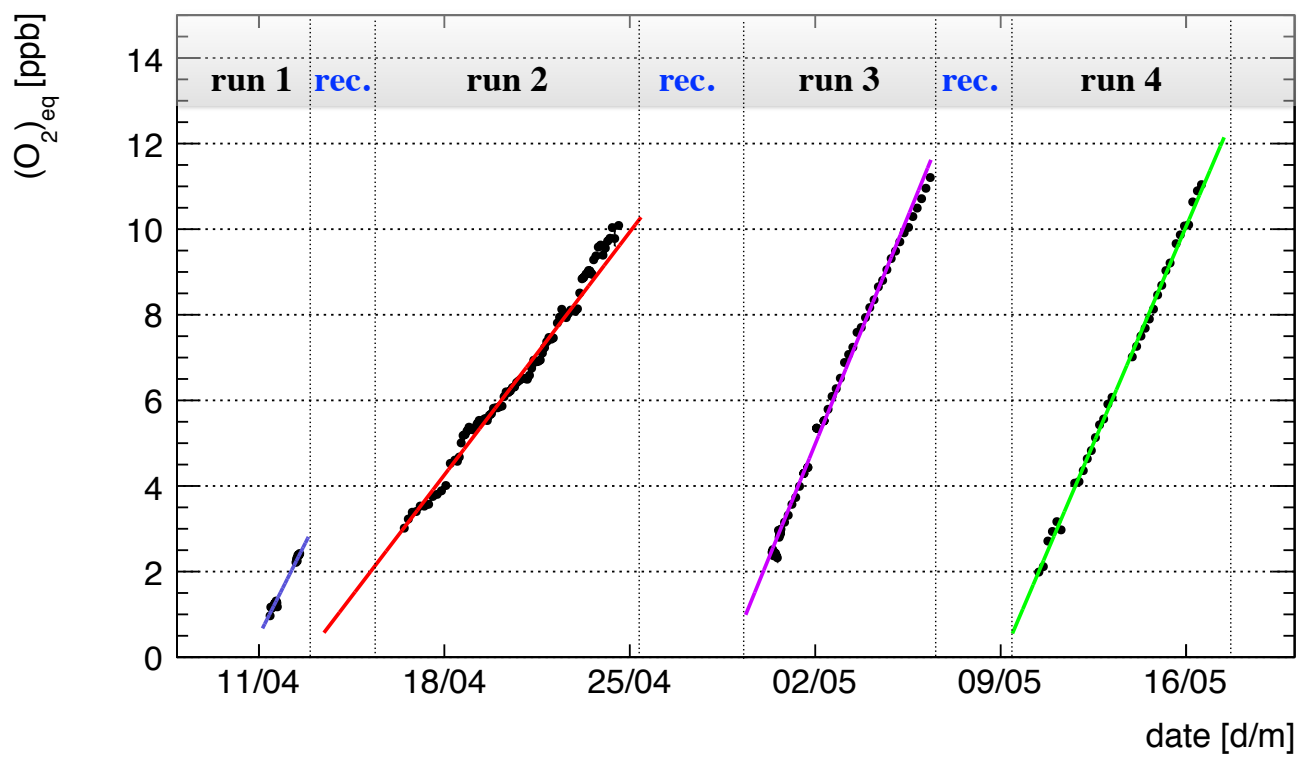

Figure 7. Evolution of the oxygen impurity during the data taking period with anode A. Linear fits to the measured points give purity losses of about $1.4 \mathrm{ppb}$ per day.

The evolution of the measured ionisation charge per unit length from view $0\left(\Delta Q_{0} / \Delta s_{0}\right)$ over the entire period is shown in Figure 8 . The mean $\Delta Q_{0} / \Delta s_{0}$ shown by the green markers on Figure \&, is proportional to the effective gain (see Eq. 3.1), and is seen to stabilise after an initial decrease. The observed gain decrease is attributed to the electrostatic charging up of the dielectric medium of the LEM during operation. For long-term operation, the gain stabilises to the value defined as $G_{\infty}$. The gain variations as a function of the gas density (i.e. related to the variations of the pressure and 


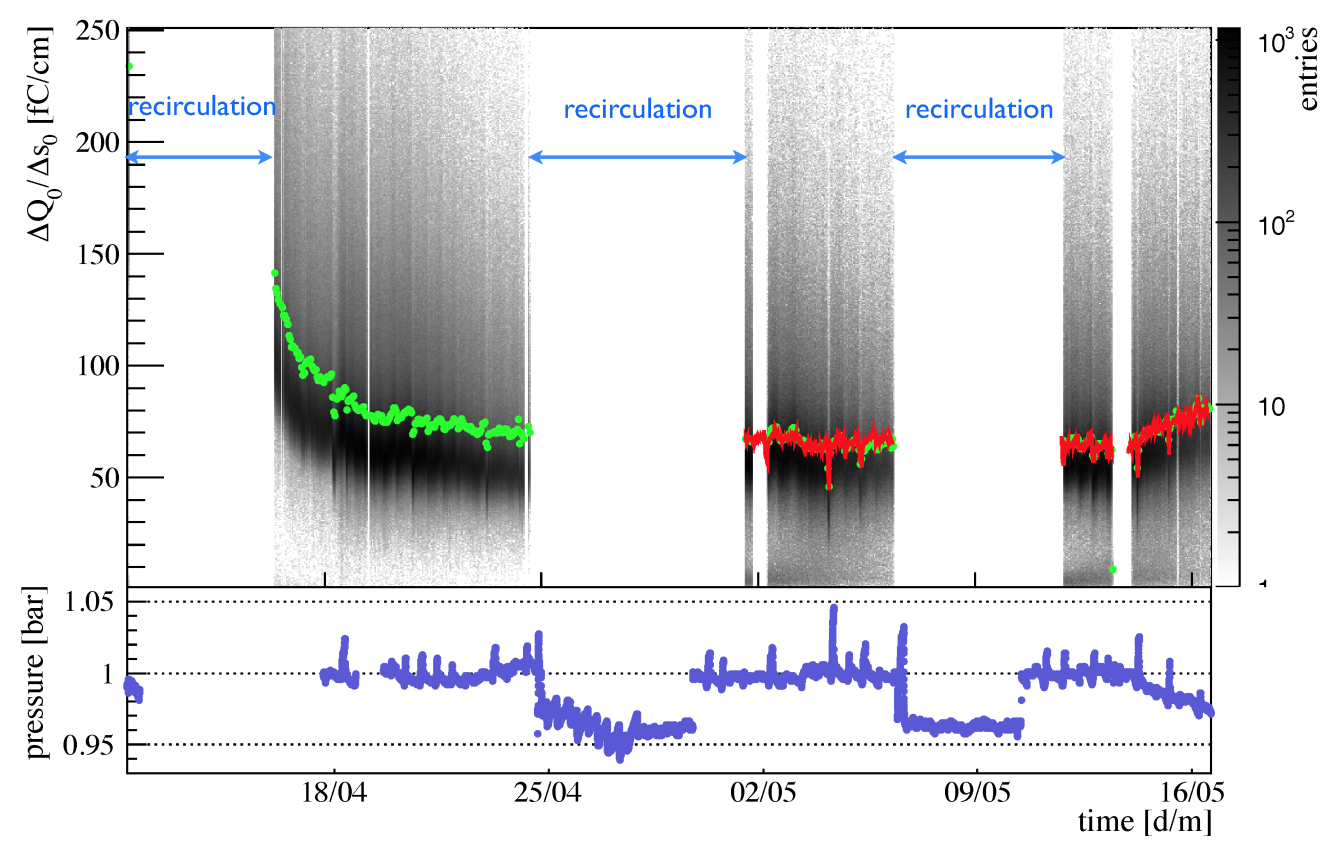

Figure 8. Evolution of the collected charge per unit length on view $0\left(\Delta Q_{0} / \Delta s_{0}\right)$ over the duration of the run. The green markers indicate the mean $\Delta Q_{0} / \Delta s_{0}$ values and the monitored pressure of the gas is shown on the bottom plot. The expected fluctuations of the gain resulting from pressure variations are well described by Eq. 3.2. (in red).

the temperature of the setup) are well described by the estimated value of Eq. 3.2, computed using the monitored pressure and a constant LEM electric field, as shown by the red curve in Figure 8 .

In order to quantitatively estimate the electrostatic charging up time of the system, the three purification periods, during which no electric field was applied across the LEM are removed from the graph and the time is offset to the start of the data taking $\left(t_{0}\right)$. A fit to the data points with Eq. 3.4 gives a gain which stabilises at $G_{\infty} \approx 15$ after an initial decrease with a characteristic time of $\tau \approx 1.6$ days.

Gain variations resulting from pressure changes are also well fitted by the function. As mentioned above, the pressure rapidly increased in our setup when the liquid Argon level in the surrounding open bath dropped below the inner vessel top flange. It was therefore necessary to constantly re-fill the outer bath with liquid Argon to maintain a stable pressure inside the detector. Nevertheless the measured charge per unit length can be corrected offline for pressure variations which allows to compute the effective gain independently of the pressure as shown in Figure 9 .

As indicated on Figure 9 by the arrow "flushed gas" at one point during the run, the gas inside the chamber was pumped out. This procedure has the effect of removing nitrogen traces $n$ the vapour phase and other contaminants that are not removed by the getter. Since this operation had no impact on the gain, we could rule out the possibility that the initial gain degradation was due to impurities slowly diffusing from the liquid into the gas phase that were not trapped by the purification cartridge.

Once the gain stabilises, switching off the electric fields for a limited period of time (e.g. 


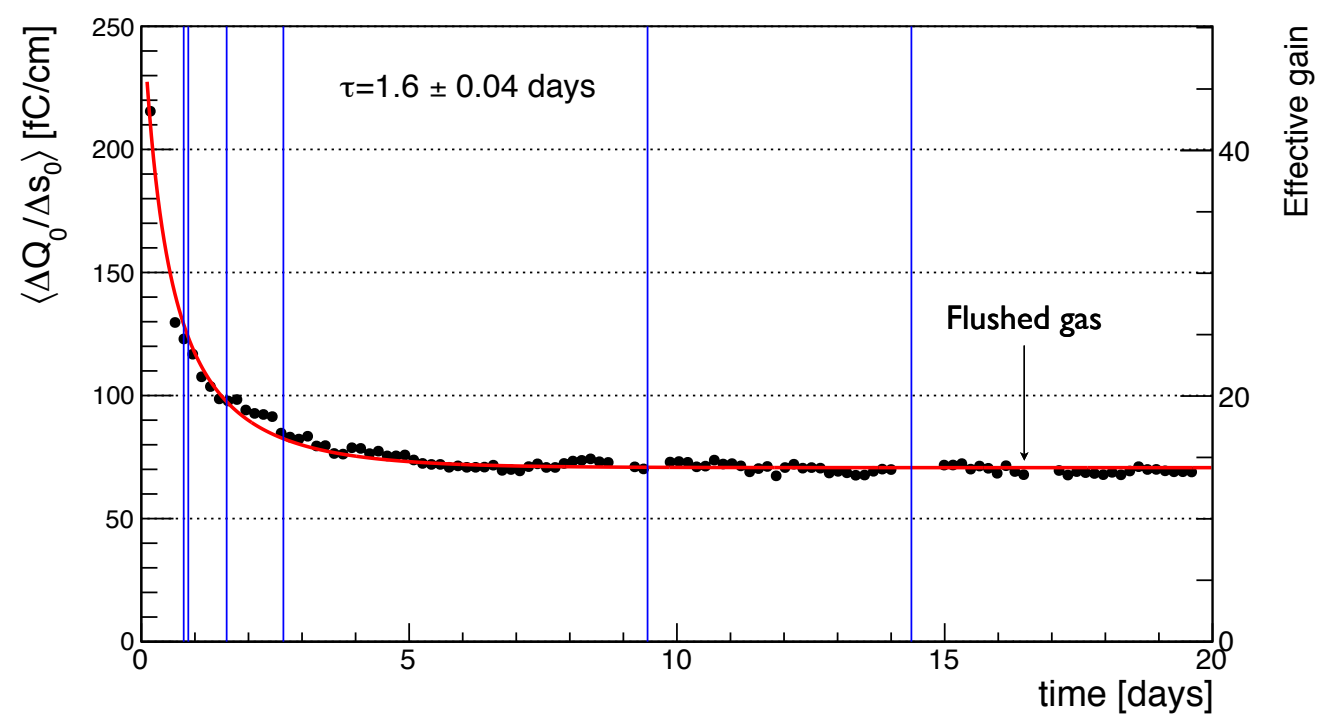

Figure 9. Evolution of the effective gain corrected for pressure variations (all data have been reported with Eq. 3.2 to a pressure of 1 bar). The data points are fitted with the function $G_{\infty} \times \frac{1}{1-e^{-t / \tau}}$. The blue lines indicate the times at which discharges occurred.

during the argon purification) does not promptly restore the value of the initially reached gain, and the gain stays at $G_{\infty}$. This is compatible with the hypothesis of the charging up of the LEM dielectric. Likely, the charges stick on the dielectric even in absence of the electric field, and argon vapour, inert and not electronegative, is not able to attach charges and remove them from the surface.

During the data-taking with anode A, six discharges occurred across the LEM, they are indicated by blue lines in Figure 9. While it is clear on the figure that those discharges do not disturb the evolution of the overall gain, locally the gain is affected in the region where the discharge occurred. This is depicted in Figure 10 where $\left\langle\Delta Q_{0} / \Delta s_{0}\right\rangle$ as a function of the reconstructed $x$ and $y$ coordinate is shown. Once a discharge occurred across a LEM hole, the gain at $t_{0}$ is recovered over a region of about $1 \mathrm{~cm}^{2}$ around the hole. As shown in Figure 11 the locally recovered gain then decreases with a similar time constant of one and a half days which supports the hypothesis that the effective gain reduction is a consequence of the charge accumulation on the dielectric of the LEM. The discharge locally removes the accumulated charges and the area then exhibits a higher gain immediately after, until charges accumulation restores the stable effective gain. This behaviour could be reproducibly observed at each discharge of the LEM. This leads us to believe that the interpretation that the electrostatic charging up of the LEM leads to the observed decrease of the effective gain, is adequate.

The signal-to-noise ratio for minimum ionising tracks is defined as the mean amplitude of the waveforms produced by the cosmic tracks divided by the average value of the noise RMS. Given our noise value of about 4-5 adc counts RMS and with the effective gain $G_{\infty} \sim 15$, the chamber is in a stable operation mode with a $S / N \sim 60$ for minimum ionising particles.

At the very beginning of the data taking with anode A, before the gain had stabilised, (i.e 

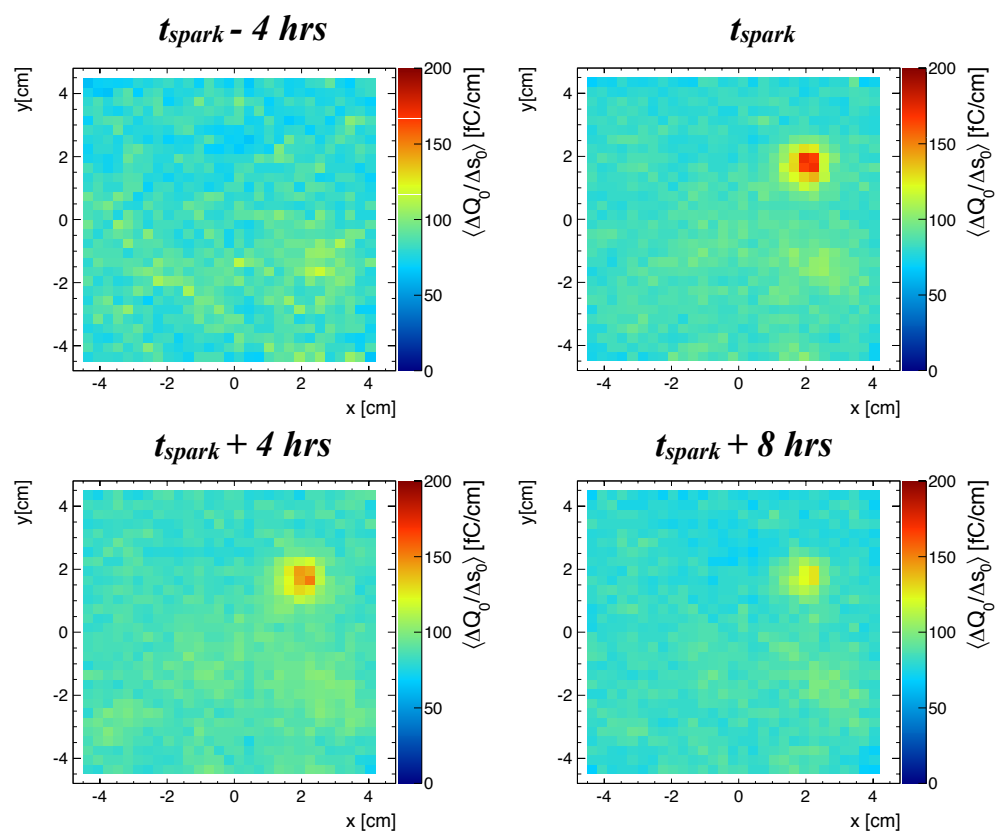

Figure 10. Time evolution of $\left\langle\Delta Q_{0} / \Delta s_{0}\right\rangle$ as a function of the $x-y$ coordinates. The plots are computed in intervals of four hours from top-left to bottom-right. A discharge occurred immediately before the second plot.

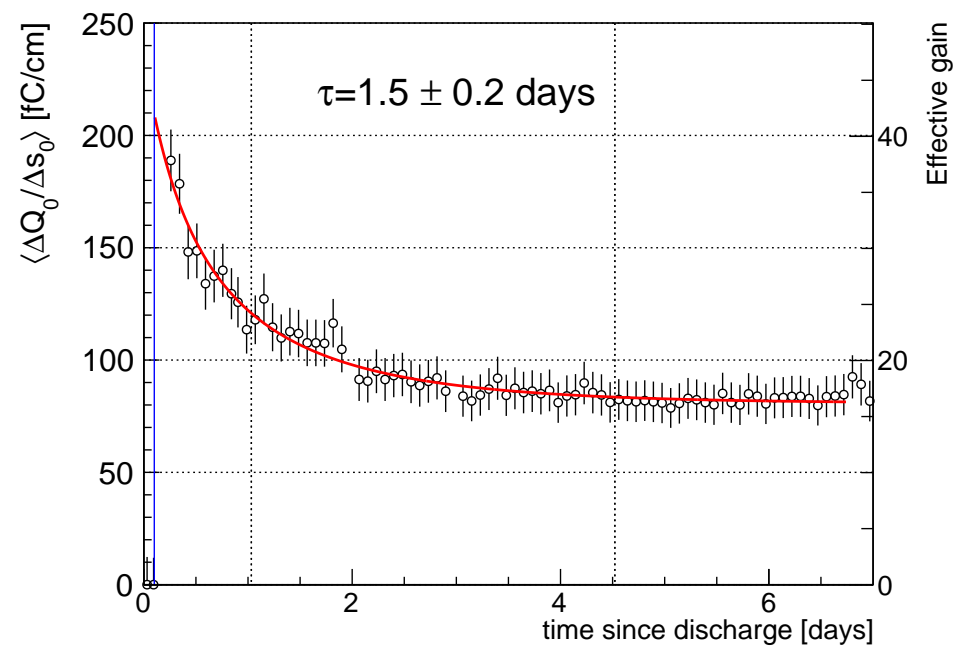

Figure 11. Evolution of the maximum $\left\langle\Delta Q_{0} / \Delta s_{0}\right\rangle$ amplitude in a $1 \mathrm{~cm}^{2}$ area around the LEM hole where the discharge occurred.

beginning of run 1 in Figure (7) we briefly tested the response of chamber in terms of effective gain for various electric fields applied across the LEM while keeping the others at the values listed in Table 3. We achieved a maximum effective gain of $G_{\text {eff }} \sim 90$ by ramping the amplification field up to $35 \mathrm{kV} / \mathrm{cm}$. The field was increased further until the breakdown voltage of $36 \mathrm{kV} / \mathrm{cm}$ was 
reached (sparks occurred across the LEM). A gain of 90 corresponds to a signal-to-noise ratio $S / N$ of about 400 for minimum ionising particles, or $S / N \approx 10$ for an energy deposition of $15 \mathrm{keV}$ on a single readout channel. Further studies will be performed to check whether the chamber can be continuously operated at larger LEM fields and if higher gains in stable conditions can be reached.

\section{Results with anodes $B, C$ and $D$}

The results reported previously were from data taken with anode A. In this section we compare data collected from four independent runs each performed with one of the anodes presented in Section 2.1. All the runs were operated at the same electric field configuration presented in Table 3. For the chosen electric fields (same as those set for anode A), the gain systematically exhibited the initial decrease reported in the previous section and stabilised at a value $G_{\infty} \sim 15$, supporting the interpretation that this behaviour is originating in the LEM and is independent of the anode. The data used to characterise the anodes is corrected for pressure variations and was collected once the gain was stable.

In Figure 12 we show relevant distributions that illustrate the performance of each anode. The left plot shows the collected charge per unit length on view $0\left(\Delta Q_{0} / \Delta s_{0}\right)$ as a function of the track azimuthal angle $\phi$, which corresponds to the angle at which the tracks cross the readout strips (see Figure 6). Distributions corresponding to the projections of the $\Delta Q_{0} / \Delta s_{0}$ in three specified $\phi$ intervals are shown in the middle. The right plot gives the resolution of the distributions in all three angular intervalls for both views. The resolution is obtained by fitting the distributions with a Gaussian convoluted Landau function and is defined as $\sigma_{\text {gauss }} /\left\langle\Delta Q_{0} / \Delta s_{0}\right\rangle$.

For all the anodes the $\Delta Q_{0} / \Delta s_{0}$ distributions are centered around $\left\langle\Delta Q_{0} / \Delta s_{0}\right\rangle \approx 75 \mathrm{fC} / \mathrm{cm}$ demonstrating the reproducibility of the detector operation with a stable gain around 15 . The values of the resolution between both views are in general symmetric around $\phi=45^{\circ}$ which is reasonable considering that the set of readout strips belonging to one view is rotated by $90^{\circ}$ with respect to the other. This also illustrates that, with the exception of anode $\mathrm{A}$, both views are completely $x-y$ symmetric. For anodes $\mathrm{A}, \mathrm{C}$ and $\mathrm{D}$ the $\Delta Q_{0} / \Delta s_{0}$ distributions are close to a Landau function as expected from the fluctuations of the collected charge per unit length. The shape of those distributions are also similar for all angular intervals. For anode B however the distributions are clearly much wider and their shapes are dependent of the angle at which the track crosses the strips. As explained in Section 2.1 anode B has only one copper track per strip which means that, for a crossing muon depositing a constant charge per unit length, neighbouring strips may not collect the same amount of charge. Charge is not lost, but the sharing locally lacks uniformity between the two views. The coarse track pitch of anode B therefore introduces large variations on the signals between neighbouring strips and consequently important fluctuations on the distributions of collected charge per unit length. This effect is illustrated in Figure 13 where two similar cosmic muon events are shown, one is acquired with anode $\mathrm{B}$ and the other with anode $\mathrm{C}$. Anode $\mathrm{C}$ has three copper tracks per strip or equivalently a track pitch of $1 \mathrm{~mm}$. It is clear from the amplitudes of the waveforms that the charge collected on anode B differs rather strongly from strip to strip. The direct consequence of those large variations is a degradation on the resolution of the collected charge per unit length. 
Anode $A$
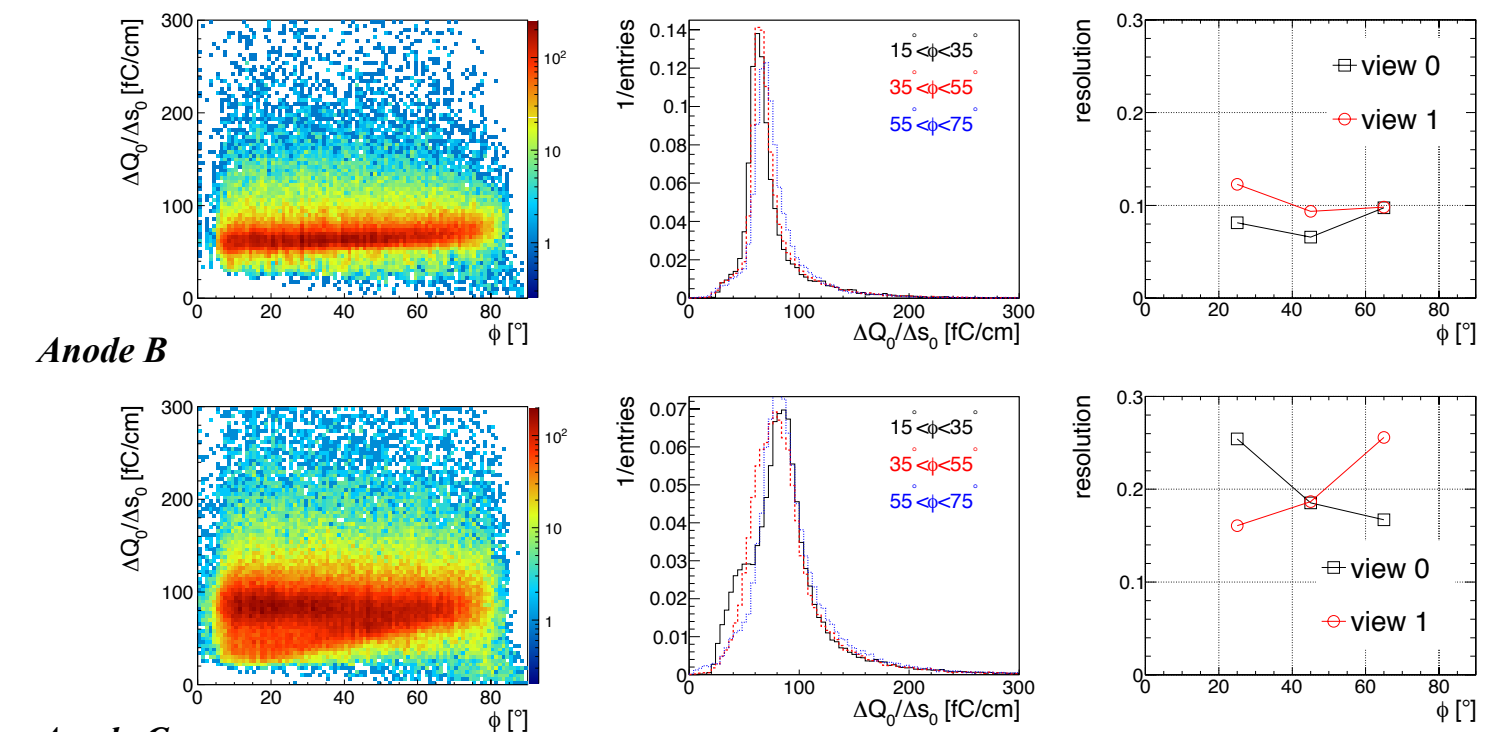

Anode C

$\phi\left[^{\circ}\right]$
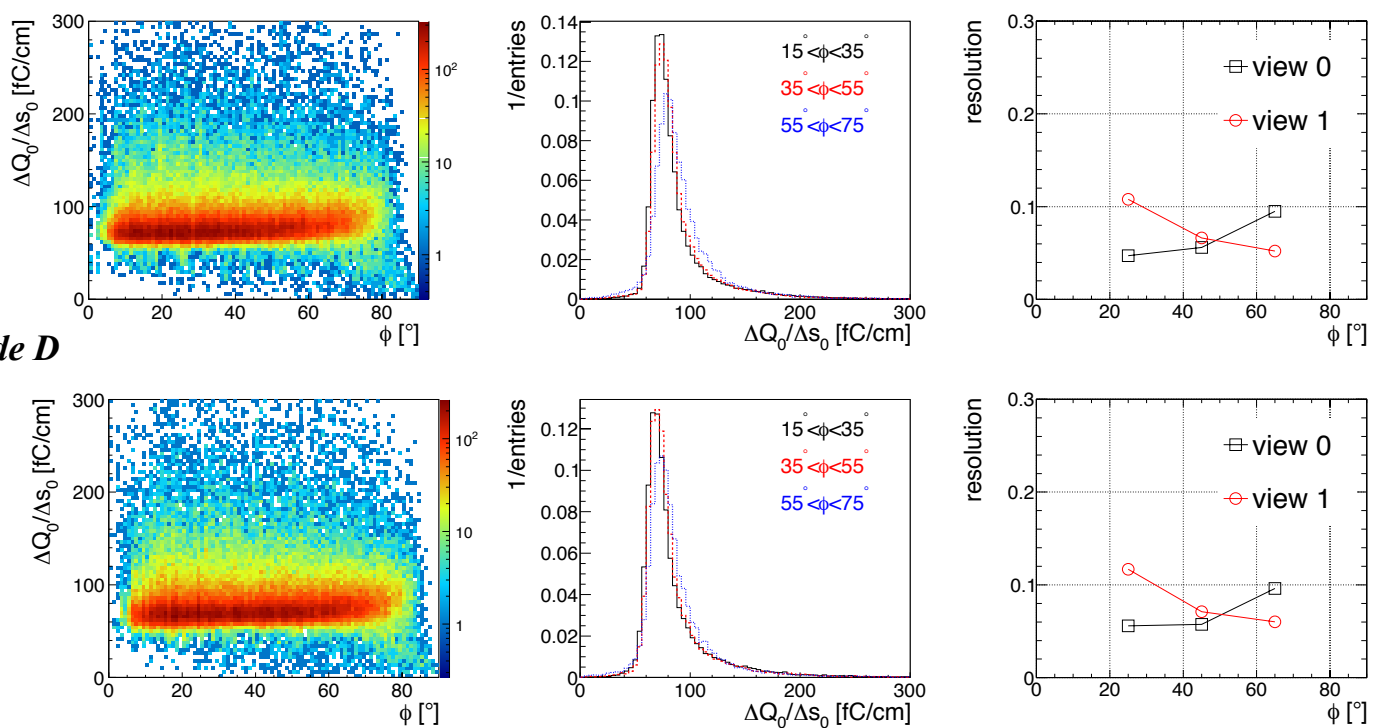

Figure 12. Charge deposition measured on view $0\left(\Delta Q_{0} / \Delta s_{0}\right)$ as a function of the track angle $\phi$ (left) and projection of the $\Delta Q_{0} / \Delta s_{0}$ distribution in three $\phi$ intervals (middle). The right plot shows the resolutions of those $\Delta Q_{0} / \Delta s_{0}$ distributions as a function of $\phi$ for both views (see text for the definition of the resolution and of the angle $\phi$ ).

\section{Summary of results}

In Table 7 the properties of each anode are summarised. For comparison those from the Kapton foil anode are also presented in the table where the values are either taken or computed from the data described in [15]. Besides the other parameters, it is important to verify that the charge is equally shared between both views. For that matter we define the asymmetry factor as the distribution of the difference between the charge collected on both views normalised to their sum. The mean and 


\section{Anode B}
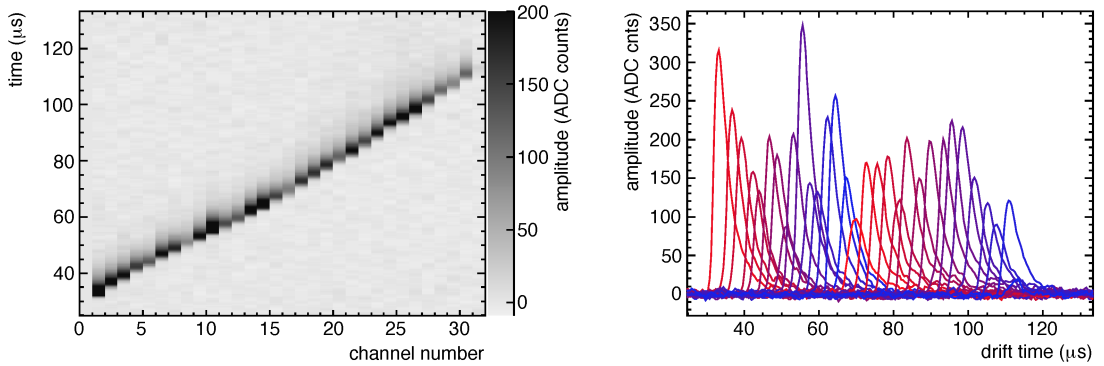

Anode C
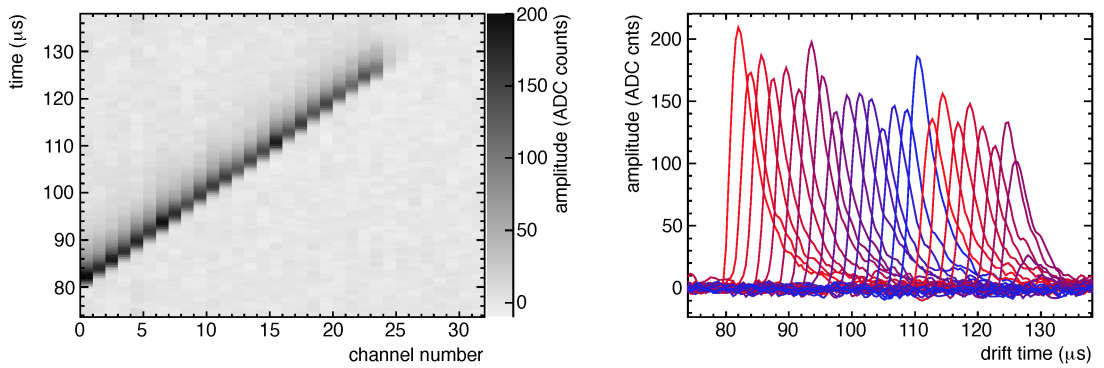

Figure 13. Two similar cosmic muon events acquired with anode $B$ and anode $C$ (only view 0 is shown). Because of the coarser track pitch of anode B, the collected charge varies significantly between neighboring strips. As a result the amplitudes of the waveforms have large variations.

RMS of those distributions are reported in Table 4 . All the anodes show good charge sharing with

\begin{tabular}{|c|c|c|c|c|c|c|}
\hline \multirow{2}{*}{\multicolumn{2}{|c|}{ anode }} & \multirow[t]{2}{*}{ capacitance $[\mathrm{pF} / \mathrm{m}]$} & \multicolumn{2}{|c|}{$\Delta Q_{i} / \Delta s_{i}$ resolution [\%] } & \multicolumn{2}{|c|}{ Asymmetry $[\%]$} \\
\hline & & & view 0 & view 1 & mean & RMS \\
\hline \multirow[t]{4}{*}{ multilayer PCB } & A & 230 & 9.3 & 11.3 & 2.1 & 8.8 \\
\hline & $\mathrm{B}$ & 100 & 22.9 & 22.4 & 0.9 & 18 \\
\hline & $\mathrm{C}$ & 260 & 5.5 & 6.4 & 0.6 & 9.8 \\
\hline & $\mathrm{D}$ & 140 & 6.7 & 7.9 & 0.7 & 9.0 \\
\hline Kapton foil & & 600 & 6.2 & 8.1 & 2.5 & 20 \\
\hline
\end{tabular}

Table 4. Summary of the results obtained for all the tested anodes. The data used for the Kapton foil anode is taken from [15].

a mean asymmetry around the percent level. Charge sharing is slightly worse for anode A which is not perfectly symmetric in the $x$ and $y$ coordinates (see Section 2.1). Anode B which benefits from a low capacitance per unit length suffers from a poor resolution on the energy loss measurement for the reasons described previously. Final designs of large area readouts will be motivated by using anodes with low capacitance per unit length which still provide the best possible resolution on the energy loss measurements. From that perspective anode D shows the best performances. 


\section{Conclusion}

We have successfully tested the characteristics of novel designs of 2D readout anodes manufactured from a single multilayer printed circuit board. Since they are robust and relatively easy to produce, they should be well suited for large area readouts. Another key feature is that the readout strips have a typical capacitance per unit length below $200 \mathrm{pF} / \mathrm{m}$ which means that the anode can be scaled to the square meter level without compromising the signal-to-noise ratio. In our effort to further simplify the design, we have shown that the electrons can be efficiently extracted from the liquid to the gas phase by means of a single grid placed in the liquid. The LEM is placed just above the liquid level and the extraction field is provided by the LEM-grid system.

A chamber newly equipped with the printed circuit board anode and the single extraction grid has achieved a high level of performance: in order to study the long-term stability, we operated the detector for a total duration of 46 days. We reproducibly observe that after an initial decrease with a characteristic time of $\tau \approx 1.6$ days, the observed gain is stable. A stable effective gain (corrected for pressure variations) of $\sim 15$ was observed and 14.6 millions trigger collected. For the first time, a maximum gain of 90 was reached with an impressive $S / N \simeq 400$ for minimum ionising particles.

Future readout planes for the GLACIER design, as proposed for instance for LBNO [5], would consist of individual square meter modules encompassing the extraction grid, the LEM and the low capacitance anode. The good quality of the data presented in this paper is therefore encouraging for future plans to assemble and test such square meter modules.

\section{References}

[1] S. Amerio et al., "Design, construction and tests of the ICARUS T600 detector" Nucl. Instrum. Meth. A527, 329-410 (2004).

[2] A. Rubbia, "Experiments for CP violation: A Giant liquid argon scintillation, Cerenkov and charge imaging experiment?," [hep-ph/0402110].

[3] A. Rubbia, "Underground Neutrino Detectors for Particle and Astroparticle Science: The Giant Liquid Argon Charge Imaging ExpeRiment (GLACIER),” J. Phys. Conf. Ser. 171, 012020 (2009). [arXiv:0908.1286 [hep-ph]].

[4] A. Badertscher et al., "Giant Liquid Argon Observatory for Proton Decay, Neutrino Astrophysics and CP-violation in the Lepton Sector (GLACIER)," [arXiv:1001.0076 [physics.ins-det]].

[5] A. Stahl, C. Wiebusch, A. M. Guler, M. Kamiscioglu, R. Sever, A. U. Yilmazer, C. Gunes and D. Yilmaz et al., "Expression of Interest for a very long baseline neutrino oscillation experiment (LBNO)," CERN-SPSC-2012-021.

[6] A. Badertscher, A. Curioni, U. Degunda, L. Epprecht, A. Gendotti, S. Horikawa, L. Knecht and D. Lussi et al., "First operation and performance of a 200 lt double phase LAr LEM-TPC with a 40x76 cm² readout,” JINST 8, P04012 (2013) [arXiv:1301.4817 [physics.ins-det]].

[7] A. Badertscher et al., "First operation of a double phase LAr Large Electron Multiplier Time Projection Chamber with a 2D projective readout anode” Nucl. Instrum. Meth. A641, 48-57 (2011).

[8] A. Badertscher et al., "Stable operation with gain of a double phase Liquid Argon LEM-TPC with a 1 mm thick segmented LEM,” J. Phys. Conf. Ser. 308 (2011) 012016. [arXiv:1010.2482 [physics.ins-det]]. 
[9] A. Badertscher, L. Knecht, M. Laffranchi, D. Lussi, A. Marchionni, G. Natterer, P. Otiougova and F. Resnati et al., "Operation of a double-phase pure argon Large Electron Multiplier Time Projection Chamber: Comparison of single and double phase operation," Nucl. Instrum. Meth. A 617, 188 (2010) [arXiv:0907.2944 [physics.ins-det]].

[10] A. Badertscher et al., "Construction and operation of a Double Phase LAr Large Electron Multiplier Time Projection Chamber," [arXiv:0811.3384 [physics.ins-det]].

[11] A. Bondar et al., "Thick GEM versus thin GEM in two-phase argon avalanche detectors," JINST 3, P07001 (2008) [arXiv:0805.2018 [physics.ins-det]].

[12] V. Boccone et al. [ The ArDM Collaboration ], "Development of wavelength shifter coated reflectors for the ArDM argon dark matter detector," JINST 4, P06001 (2009). [arXiv:0904.0246 [physics.ins-det]].

[13] A. Buzulutskov, "Advances in Cryogenic Avalanche Detectors (review)," JINST 7, C02025 (2012) [arXiv:1112.6153 [physics.ins-det]].

[14] V. Chepel and H. Araujo, "Liquid noble gas detectors for low energy particle physics," JINST 8, R04001 (2013) [arXiv:1207.2292 [physics.ins-det]].

[15] F. Resnati, "Modeling, design and first operation of the novel double phase LAr LEM-TPC detector", $\mathrm{PhD}$ thesis, ETH Zurich (2012)

[16] D. Lussi, "Study of the response of the novel LAr LEM-TPC detector exposed to cosmic rays and a charged particle beam", PhD thesis, ETH Zurich (2013)

[17] D.G. Cassel and H. Kowalski, "Pattern recognition in layered track chambers using a tree algorithm" Nucl. Instrum. Meth. A185, 235-251 (1981).

[18] J. Rico, "First study of the stopping Muon sample with the ICARUS T600 detector," Ph.D. Dissertation, ETH 14906 (2002)

[19] S. Amoruso et al. [ICARUS Collaboration], "Study of electron recombination in liquid argon with the ICARUS TPC,” Nucl. Instrum. Meth. A 523, 275 (2004).

[20] T. Aoyama, "Generalized Gas Gain Formula for Proportional Counters", Nucl. Instrum. Meth. A234, 125 (1985).

[21] R. Veenhof., "Magboltz - transport of electrons in gas mixtures", version 8.9, http://consult.cern.ch/writeup/magboltz/

[22] A.F. Borghesani et al. "Electron transmission through the Ar liquid-vapor interface," Phys. Lett. 149, 481-484, (1990). 